As seen from the graphs, the curves which best fit our values of $R_{T} / R_{0}{ }^{\circ} \mathrm{C}$. are straight lines parallel to the temperature axis. The absolute experimental error in the ratios we estimate as not greater than $0 \cdot 00002$. According to the data of Meissner and Voigt, we should expect an increase in $R_{T} / R_{0}{ }^{\circ} \mathrm{C}$. of between 0.00007 and 0.00014 in the temperature interval $4.2^{\circ}$ to $2 \cdot 2^{\circ} \mathrm{K}$. Since Nos. 5 and 6 show smaller values of $R_{T} / R_{0^{\circ} \mathrm{C}}$, in this region than any previously reported for aluminium, we conclude that, within the accuracy of our measurements, there is no increase in the resistance of pure aluminium down to $2 \cdot 2^{\circ} \mathrm{K}$.

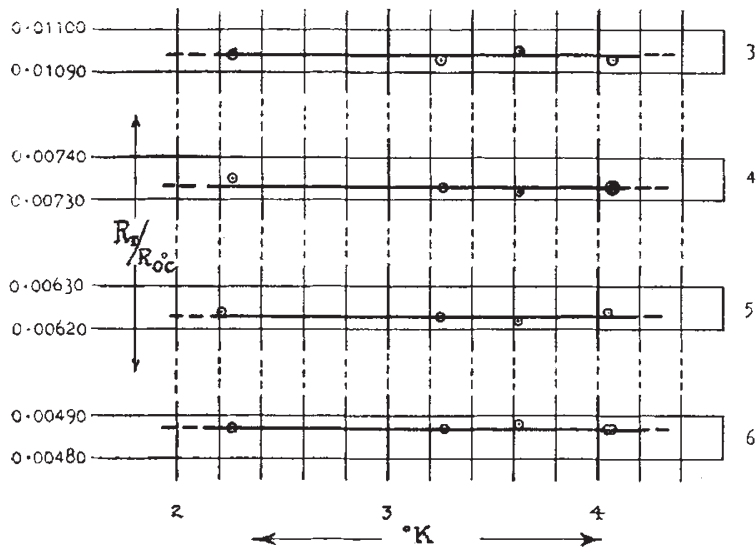

FIG. 1. Relation of $R_{T} / R_{0}{ }^{\circ} \mathrm{c}$. to temperature for aluminium at low temperature.

The liquid helium for these experiments was produced by the new helium liquefier designed by Prof. P. Kapitza ${ }^{5}$ A detailed report of these experiments will appear elsewhere.

H. A. Boonse.

H. NiewodNICZański.

Royal Society Mond Laboratory, Cambridge. April 23.

${ }^{1}$ Leiden Comm., No. 181 ; 1926.

Ann. Phys., 7, 761 ; 1930.

Leiden Comm, No, 224c: 1933.

-W. J. de Haas, J. de Boer and G. J. van den Berg, Physica, 1, 1115 ; 1934.

Proc. Roy. Soc., A, 147, $189 ; 1934$.

\section{Range of Action of Surface Forces}

IN a recent letter, Wilsdon, Bonnel and Nottage ${ }^{1}$ suggest that anomalies observed in the vapour pressure, osmotic pressure and flow of water in porous materials and in capillaries are due to oriented chains of water molecules extending to a distance of $50 \times 10^{-5} \mathrm{~cm}$. from the surface. The possibility of such a structure has been suggested by Hardy ${ }^{2}$, and by Watson and Menon ${ }^{3}$, who found that a polished plate floated in air or water at a height of $40 \times 10^{-5}$ $\mathrm{cm}$. from a parallel plate. Further experiments", however, carried out at Sir William Hardy's suggestion showed that the separation of the plates was due to dust or to some similar commonplace cause.

More recently, Derjaguin', from observations on the resistance offered by water to the movement of an oscillating lens, concluded that a water film $1 \times 10^{-5} \mathrm{~cm}$. thick has a rigidity about $1 / 300$ of that of solid lead. We have measured the resistance offered to the flow of thin liquid films enclosed between parallel surfaces. A relatively thick film $\left(60 \times 10^{-5} \mathrm{~cm}\right.$.) of a 1 per cent solution of ammonium oleate in water (a liquid crystal known to possess a slight bulk rigidity) gave the results shown in Fig. 1 (Curve 1), a pressure head of about $2 \mathrm{~cm}$. being required to cause appreciable flow.

A water film, $16 \times 10^{-5} \mathrm{~cm}$. thick, gave Curve II, which is linear and passes through the origin. Similar results were obtained with alcohol, cyclohexane,

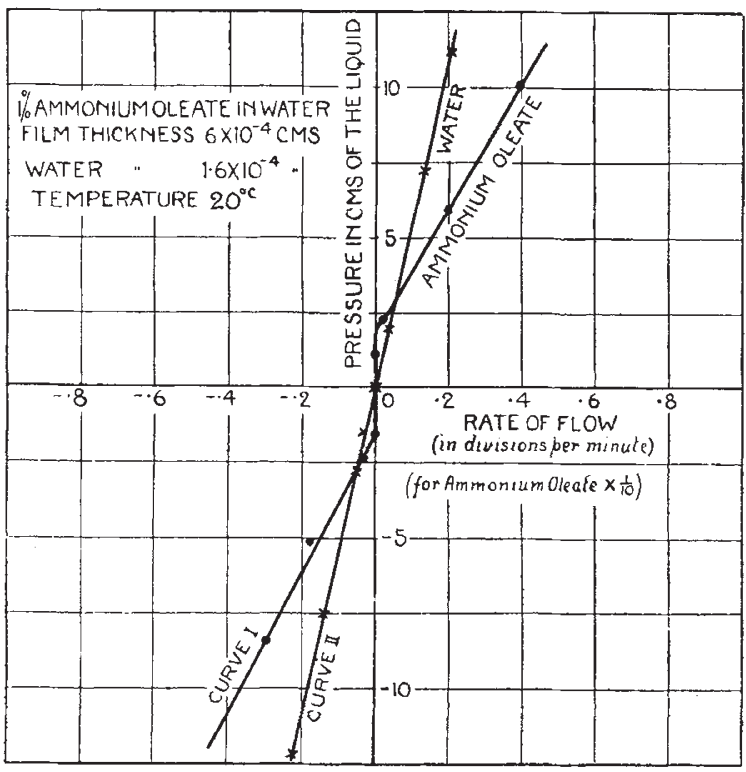

FIG. 1. Resistance to flow of thin liquid flims between parallel surfaces

acetic acid and ethyl palmitate. Experiments with thinner films (circa $10^{-5} \mathrm{~cm}$.), and with liquids cooled to within $0.1^{\circ} \mathrm{C}$. of the freezing point, also gave no evidence of rigidity, since the smallest measurable pressure $(<1 \mathrm{~mm}$. head) always produced a normal flow and within the accuracy of the measurements, the viscosity of the film was the same as that of the liquid in bulk.

The mechanical properties of the first few molecular Jayers of an adsorbed gas or liquid may be profoundly modified by the solid surface, but no such effects can be detected at distances of $10^{-5} \mathrm{~cm}$. and the extension of rigid chains to a distance of $50 \times 10^{-5}$ $\mathrm{cm}$. seems quite out of the question.

F. P. Bowden.

S. H. Bastow.

Laboratory of Physical Chemistry, Cambridge. March 12.

1 Wilsdon, Bonnel and Nottage, Nature, 135, 186, Feb. 2, 1935.

Hardy, Phil. Trans. Roy. Soc., A, 230, 1 ; 1932

Watson and Menon, Proc. Roy. Soc., A, 118, 2111928.

- Bastow and Bowden, Proc. Roy. Soc., A, 134, 104 ; 1931.

Derjaguin, Z. Phys., 84, 657; 1933.

\section{Interchange of Heavy Atoms in Organo-Metallic Methyls}

USING the radioactive indicator method in which radioactive isotopes are used to indicate transference of atoms, we have obtained evidence which suggests that both lead and bismuth, deposited, presumably as oxides, on a metallic surface, can exchange with lead in lead tetra-methyl and bismuth in $\mathrm{Bi}\left(\mathrm{CH}_{3}\right)_{3}$ in ether solution at room temperature.

For bismuth the radioactive isotopes radium $\mathbf{E}$ and thorium $\mathrm{C}$, periods 5 days and 60.5 minutes respectively, were used, and for lead, ThB with period $10 \cdot 6$ hours was employed. 